\title{
ESCALA DE EMPATIA FOCADA EM GRUPOS: EVIDÊNCIA PSICOMÉTRICA EM DIFERENTES INSTITUIÇÕES SÓCIO-EDUCACIONAIS
}

\begin{tabular}{l}
\hline SCALE OF EMPATHY FOCUSED ON GROUPS: PSYCHOMETRIC EVIDENCE IN \\
DIFFERENT EDUCATIONAL INSTITUTIONS
\end{tabular}

DOI: 10.22199/S07187475.2016.0001.00002

Recibido: 23 de noviembre del 2015 | Aceptado: 29 de marzo del 2016

NILTON S. FORMIGA 1 ; MIRIANE DA S. SANTOS BARBOZA 2 ; CLEONICE P. DOS S. CAMINO 3

(FACULDADE INTERNACIONAL DA PARAíba/LAUREATE INTERNATIONAL UNIVERSitIES, João Pessoa, PB, Brasil)

\begin{abstract}
RESUMO
A empatia focada em grupos refere-se à capacidade afetiva das pessoas sentirem e pensarem o sofrimento de grupos específicos. 0 presente artigo procurou reunir evidências da validade fatorial e consistência interna da Escala de Empatia focada em Grupos (EEG), considerando amostras distintas de jovens. 376 estudantes do ensino médio, entre 17 e 33 anos, da cidade de João Pessoa-PB, sendo 200 de uma instituição pública e 176 de uma instituição militar de ensino, responderam a EEG e a um questionário demográfico. Observou-se que, para ambas as amostras, a escala revelou indicadores psicométricos que garantem a estrutura multifatorial já encontrada por outros autores. Ainda se constatou que os jovens militares apresentaram médias superiores à dos jovens civis em relação a três de quatro componentes testados, mostrando-se mais empáticos ao sofrimento de grupos minoritários, ao de apenados e ao de trabalhadores do que os estudantes civis.
\end{abstract}

PALAVRAS-CHAVE: Empatia, avaliação, modelagem estrutural, jovens civis e militares.

\section{ABSTRACT}

Introduction: Empathy focused on groups is concerned with humans' capacity to feel and think about other people's pain and sorrow. Objective: This article aims at gathering evidence of factorial validity and internal consistency of the Empathy Scale focused on Groups (ESG) considering varied samples of young people. Method: 376 high school students from the city of João Pessoa - Paraíba, (200 from public institutions and 176 from a military institution), between the ages of 17 and 33, answered the ESG and a demographic questionnaire. Results: In both samples, the scale revealed psychometric evidence that confirms the existence of a multifactorial structure already identified in previous research. It has also been found that the young military achieved higher averages than the young civilians regarding three or four aspects of the test. Conclusion: Young military students are more sympathetic with the suffering of minority groups (prisoners and workers) than young civilian students.

KEY WORDS: Empathy, assessment, structural modeling, young civilians and young military.

\footnotetext{
1. Doutorado em Psicologia Social pela Universidade Federal da Paraíba. Atualmente é professor Faculdade Internacional da Paraíba/Laureate International Universities. Endereço para correspondência: Rua Lionildo Francisco de Oliveira, 380. Bairro dos Estados. CEP.: 58030-216 João Pessoa - PB. Brasil. E-mail: nsformiga@yahoo.com 2. Doutorado em Psicologia Social pela Universidade Federal da Paraíba, Professora da Universidade Estadual do Piauí. E-mail: minianesantos@hotmail.com

3. Doutorado em Psicologia pela Université Catholique de Louvain (Bélgica), professora da Pós-graduação em Psicologia Social da Universidade Federal da Paraíba. E-mail: cleocamino@yahoo.com.br
} 
INTRODUÇÃO

A investigação sobre o desenvolvimento e manutenção da empatia desenvolvida pelas pessoas vem interessado quanto uma proposta de trabalho muito relevante aos pesquisadores de distintas áreas científica (Psicologia, Educação, Medicina, etc.); sobretudo, quando se pretende verificar 0 padrão atitudinal do ser humano na sociedade contemporânea em relação a sensibilidade frente as outras pessoas e 0 seu entorno sócio-ambiental. Este tema tem sido muito atual, justamente, devido a égide de práticas individualistas que envolve as pessoas e suas relações interpessoais, as quais, algumas vezes, camufladas com preocupações em relação ao outro, mas que, em alguns momentos visam mais a si mesmo do que ao outro (Dumont, 1985; Lipovetsky \& Charles, 2004; Oceja \& Jiménez, 2007). Tal momento é nomeado por Bentrán e Cardona (2005) como 0 tempo da sociedade narcísica e, excessivamente, egoísta que percorre 0 caminho inverso da episteme das relações humanas quanto a cooperação e grupalidade (Morin, 2005).

O estudo sobre a empatia não é um tema novo; já no final do século XIX se discutia a respeito desse construto na psicologia alemã, especificamente, no campo das influências fisiológicas da psicologia sensorial, nos estudos acerca da percepção, da consciência e da introspecção, bem como, nos estudos sobre a experiência estética na arte (Schultz \& Schultz, 1998; Wispé, 1990). Todos esses estudos foram influenciados, direta ou indiretamente, pela Psicologia da Gestalt.

Dentre as diferentes definições acerca da empatia, destaca-se, neste trabalho, a perspectiva de Hoffman (1991); segundo esse autor, a empatia é a capacidade que uma pessoa tem de colocar-se no lugar do outro (role-taking), inferir seus sentimentos e, a partir do conhecimento gerado por este processo, dar uma resposta afetiva mais adequada para a situação do outro do que para sua própria situação. Definida dessa forma, a empatia seria então, uma habilidade que permite ao ser humano estabelecer elos afetivos durante as interações e motiva comportamentos prósociais.

No que diz respeito à avaliação da empatia, observa-se 0 uso de uma variedade de técnicas e métodos, por exemplo: questionários, escalas, histórias com ou sem fotos, figuras, gravações, análise das expressões faciais e dos gestos, bem como, a verificação da condutibilidade e temperatura da pele e da freqüência cardíaca (Eisenberg \& Strayer, 1990). Cada uma dessas técnicas apresenta vantagens e desvantagens e elas podem ser constatadas em relação à administração de instrumentos, ao investimento econômico em aparelhos computacionais, ao desconforto das pessoas testadas, etc. (Strayer \& Eisenberg, 1987).

No sentido de minimizar as desvantagens, ligadas a algumas formas de avaliação da empatia, se sobressai o uso de instrumentos tipo papel e lápis, que têm sido um dos mais requisitados em todo mundo (Pérez-Albéniz, Paúl, Etxeberría, Montes \& Torres, 2003), não somente devido à facilidade de aplicação, mas também, em função da sua economia na produção do material e no momento da sua administração (Strayer \& Eisenberg, 1987). Dentre as escalas mais utilizadas, destacase: a Hogan Empathy Scale (HES, Hogan, 1969), 0 Questionnaire Measure of Emotional Empathy (QMEE, Mehrabian \& Epstein, 1972) e o Interpersonal Reactivity Index (IRI, Davis, 1980).

Recentemente, no Brasil, foi publicada a Escala de Empatia focada em Grupos -EEG - (Galvão, Camino, Gouveia \& Formiga, 2010), que objetiva mensurar a empatia em um nível que ultrapassa as relações interpessoais. Nesse sentido, a EEG difere da maioria dos instrumentos existentes 
acerca desta temática e destacada no parágrafo acima; a proposta dos autores da EEG foi a de criar uma medida de empatia que avaliasse a capacidade de uma pessoa empatizar com pessoas ou animais que se encontram em situações de sofrimento, decorrentes de contextos sociais, etc. Assim, a EEG difere das escalas existentes que avaliam a empatia de uma pessoa em relação a outra pessoa, cujo sofrimento não depende, necessariamente, de problemas sociais.

Galvão et al. (2010) observaram, em uma amostra de sujeitos de 14 a 19 anos, a partir de uma análise fatorial dos componentes principais, que 0 referido construto se organizava em quatro dimensões: Empatia com grupos minoritários, Empatia com animais, empatia com trabalhadores e por fim, Empatia com apenados. Essas dimensões apresentaram índices de consistência interna aceitos pela literatura vigente (por exemplo, 0,68 a 0,80, em suas dimensões) (Tabachnick \& Fidell, 2001). A fim de corroborar as dimensões, previamente, encontradas, Galvão et all. (2010), em uma segunda amostra com sujeitos de 13 a 17 anos, realizaram uma análise fatorial confirmatória e verificando a estrutura fatorial encontrada no primeiro estudo; nela, os indicadores psicométricos estiveram de acordo com o que propunha a literatura estatística (Hair, Anderson, Tatham \& Black, 2005; Joreskög \& Sörbom, 1989). Nos resultados, observaram, mais uma vez, a multifatorialidade da EEG, comprovando o que foi encontrado na análise exploratória, bem como, que os índices de consistência internar fora próximo aos já encontrado no primeiro estudo.

Uma especificidade da EEG, segundo seus idealizadores, é que ela permite verificar a empatia com animais, algo negligenciado nos estudos acerca da empatia (Paul, 2000). Em relação a este último aspecto, lembra-se aqui o que disse M. Gandhi, um dos maiores modelos de moral e compaixão: "a grandeza de uma nação e seu progresso moral pode ser julgado pela forma que seus animais são tratados". Outra contribuição da EEG, é o fato dela considerar a empatia com 0 sofrimento de apenados, algo não tão consensual. Sem uma variável dessa natureza, corre-se o risco de fazer a avaliação de uma empatia facilmente provocada, deixando de lado, a avaliação da empatia em grupos (pessoas ou coisas) que, na dinâmica social, geralmente, é colocado na periferia das relações ou tipo como algo 'com pouco significado' para a vida das pessoas e seu entorno humano.

Diante do que foi exposto sobre a EEG acredita-se que ela, de fato, poderá contribuir para a compreensão do fenômeno da empatia seguindo uma perspectiva alternativa de avaliação de variáveis. No entanto, como problematizam os criadores do instrumento, é necessário que outros estudos investiguem a sua adequação a outros contextos. É neste sentido que 0 presente estudo tem como objetivo: verificar a acurácia dessa escala com base nos indicadores psicométricas da estrutura fatorial encontrada por Galvão et al. (2010), assim como, a adequabilidade dessa estrutura com amostras de sujeitos em diferentes contextos sócio-escolares.

\section{MÉTODO \\ Participantes}

376 estudantes do ensino médio da cidade de João Pessoa-PB, Brasil, sendo 200 deles foram de uma instituição pública de ensino, de 17 a 20 anos, predominantemente do sexo feminino (54\%), e 176 deles de uma instituição militar de ensino, de 17 a 33 anos, predominantemente do sexo masculino (76\%). Esta amostra foi nãoprobabilística, isto é, de conveniência, tendo participado as pessoas que, convidadas, aceitaram colaborar. 
Instrumento

Escala de Empatia focada em Grupos (EEG) - desenvolvida por Galvão et al. (2010), é composta por 27 itens, relacionados a quatro contextos sociais: grupos minoritários (ex: negros sendo menosprezados), trabalhadores (ex: pessoas que são obrigadas a deixar 0 campo para trabalhar na cidade), presos (ex: presos vivendo em situações desumanas) e animais (ex.: animais sendo maltratados), que devem ser avaliados em uma escala de cinco pontos, variando de 1 (afeta pouco) a $\mathbf{5}$ (afeta muito).

A EEG revelou indicadores psicométricos consistentes, identificando quatro fatores (Galvão et al., 2010): empatia com grupos minoritários, empatia com animais, empatia com trabalhadores $e$ empatia com apenados; para cada um desses fatores, foram encontrados Alphas de Cronbach que variavam de 0,69 a 0,80. Considerando a Análise Fatorial Confirmatória, realizada com o AMOS 7.0, destinado aos cálculos de modelagem de equações estruturais (SEM), foi comprovado a existência dos mesmos fatores observados na análise exploratória $\left[X^{2} / \mathrm{gl}=\right.$ $1,14, \mathrm{GFI}=0,92, \mathrm{AGFI}=0,90, \mathrm{CFI}=0,98$, $\operatorname{RMSEA}(90 \% \operatorname{IC})=0,02(0,01-0,04), \mathrm{CAIC}=$ $514,59$ e ECVI $=1,37(1,26-1,55)]$.

\section{Procedimento}

Todos os procedimentos adotados nesta pesquisa seguiram as orientações previstas na Resolução 196/96 do CNS e na Resolução 016/2000 do Conselho Federal de Psicologia (CNS, 1996; ANPEPP, 2011).

\section{Administração}

Para a aplicação do instrumento, o responsável pela coleta dos dados contatou diretamente os diretores e/ou coordenadores das instituições de ensino pesquisadas. Em seguida, pediu autorização, junto aos professores, para ocupar uma aula e aplicar os questionários.
Após ser autorizado, o responsável pela coleta de dados apresentou, de forma sumária, os objetivos da pesquisa aos estudantes, os convidando para participar voluntariamente do estudo. Aos estudantes foi dito que não havia resposta certa ou errada e que eles não deveriam se sentir obrigados a responder aos questionários, podendo desistir a qualquer momento, seja quanto tivesse 0 instrumento em suas mãos ou ao iniciar a leitura, ou em outra eventual condição.

A todos foi assegurado 0 anonimato das suas respostas, enfatizando que elas seriam tratadas estatisticamente em seu conjunto. Apesar do questionário ser auto-aplicável, contando com as instruções necessárias para serem respondidos individualmente, um aplicador, previamente treinado, esteve presente para apresentar os instrumentos, para retirar eventuais dúvidas ou realizar esclarecimentos que se fizessem indispensáveis, e, para conferir a qualidade geral das respostas emitidas pelos respondentes.

\section{Análise de Dados}

A tabulação e a análise dos dados (estatísticas descritivas e ANOVA) foram efetuadas por meio do pacote estatístico SPSS - versão 21.0. Porém, para a análise fatorial confirmatória, utilizou-se o programa AMOS 21.0. Este tipo de análise permite testar hipóteses específicas sobre a estrutura latente do modelo (por exemplo, número de fatores, cargas fatoriais), apresentando os respectivos indicadores do "goodness", os quais possibilitam avaliar a qualidade de ajuste do modelo a que se propõe (Hair, Anderson, Tatham, \& Black, 2005; Tabachnick \& Fidell, 2001; Van de Vijver \& Leung, 1997), como por exemplo:

- $0 X^{2}$ (qui-quadrado) testa a probabilidade do modelo teórico se ajustar aos dados; quanto maior este valor pior o ajustamento. Este tem sido pouco empregado na literatura, sendo 
mais comum considerar sua razão em relação aos graus de liberdade $\left(x^{2} / g\right.$. I.). Neste caso, valores até 3 indicam um ajustamento adequado.

- O Goodness-of-Fit Index (GFI) e 0 Adjusted Goodness-of-Fit Index (AGFI) são análogos ao $R^{2}$ em regressão múltipla. Portanto, indicam a proporção de variância-covariância nos dados, explicadas pelo modelo. Estes variam de 0 a 1 , com valores na casa dos 0,90, indicando um ajustamento satisfatório.

- Raiz Quadrada Média Residual (RMR), que indica o ajustamento do modelo teórico aos dados, na medida em que a diferença entre os dois se aproxima de zero (Joreskög \& Sörbom, 1989).

- A Root-Mean-Square Error of Approximation (RMSEA), com seu intervalo de confiança de $90 \%$ (IC90\%), é considerado um indicador de "maldade" de ajuste, isto é, valores altos indicam um modelo não ajustado. Assume-se como ideal que o RMSEA se situe entre 0,05 e 0,08 , ou menos.

- 0 Comparative Fit Index (CFI) compara, de forma geral, o modelo estimado com 0 modelo nulo, considerando valores mais próximos de um como indicadores de ajustamento satisfatório (Hair, Anderson, Tatham \& Black, 2005).

- Tucker-Lewis Index (TLI), apresenta uma medida de parcimônia entre os índices do modelo proposto e do modelo nulo. Varia de zero a um, com índice aceitável acima de 0,90 (Bilich, Silva \& Ramos, 2006).

- O Expected Cross-Validation Index (ECVI) e o Consistent Akaike Information Criterion (CAIC) são indicadores geralmente empregados para avaliar a adequação de um modelo em relação a outro. Valores baixos do ECVI e CAIC expressam 0 modelo com melhor ajuste.

\section{RESULTADOS}

Neste estudo, a titulo de lembrança de leitor, buscou-se avaliar a estrutura da escala de empatia focada em Grupos (EEG) em duas amostras de jovens em contextos sócio-escolares distintos (jovens civis e jovens militares). Efetuaram-se, no pacote estatístico AMOS 16.0, a análise fatorial confirmatória e de modelagem de equação estrutural para as amostras coletadas; hipotetizou-se a existência de quatro fatores de acordo com a proposta de Galvão et al. (2010): Empatia com grupos minoritários, Empatia com animais, Empatia com trabalhadores e Empatia com apenados.

Deixando livre as covariâncias (phi, $\varphi$ ) entre os fatores, observaram-se que indicadores de qualidade de ajuste do modelo com a amostra geral, com a amostra dos jovens civis e com a amostra dos jovens militares, atenderam às recomendações psicométricas apresentadas na literatura a respeito dos índices (limites e intervalos) destinados a qualidade da organização fatorial proposta(Byrne, 1989, 2006; Tabachnick \& Fidell, 1996; Van de Vijver \& Leung, 1997), os quais, expressos na seção da análise dos dados no método deste estudo. Os resultados obtidos nestas análises, observados em negrito, na Tabela 1, revelam que 0 melhor modelo para a empatia focada em grupos foi 0 modelo tetrafatorial, em todas as amostras.

Além disso, observe-se que os resultados da EEG expostos na Tabela 1 apresentaram indicadores de ajustes, melhores do que os encontrados no estudo dos autores supracitados (ver Galvão et all., 2010); essa condição, vem corroborar os achados desses autores e garantir a consistência da estrutura fatorial da escala nas referidas amostras. 
TABELA 1.

Indicadores psicométricos da estrutura fatorial da escala de empatia focada em grupos em jovens de distintos contextos sócioescolares.

\begin{tabular}{|c|c|c|c|c|c|c|c|c|c|}
\hline $\begin{array}{l}\text { Modelo por } \\
\text { amostra }\end{array}$ & $\mathrm{c}^{2} / \mathrm{gl}$ & RMR & GFI & AGFI & CFI & TLI & $\begin{array}{l}\text { RMSEA } \\
\text { (intervalo) }\end{array}$ & CAIC & $\begin{array}{c}\text { ECVI } \\
\text { (intervalo) }\end{array}$ \\
\hline \multicolumn{10}{|l|}{ Modelo 1* } \\
\hline Unifatorial & 3,70 & 0,08 & 0,87 & 0,80 & 0,82 & 0,76 & 0,08 & 925,54 & 1,87 \\
\hline Tetrafatorial & 1,16 & 0,04 & 0,97 & 0,94 & 0,99 & 0,99 & $\begin{array}{c}0,02 \\
(0,00-0,03)\end{array}$ & 787,84 & $\begin{array}{c}0,87 \\
(0,80-0,93)\end{array}$ \\
\hline \multicolumn{10}{|l|}{ Modelo $2^{* *}$} \\
\hline Unifatorial & 2,72 & 0,11 & 0,84 & 0,76 & 0,75 & 0,75 & 0,09 & 793,45 & 1,94 \\
\hline Tetrafatorial & 1,03 & 0,06 & 0,94 & 0,91 & 0,99 & 0,99 & $\begin{array}{c}0,02 \\
(0,00-0,03)\end{array}$ & 565,73 & $\begin{array}{c}1,36 \\
(1,34-1,53)\end{array}$ \\
\hline \multicolumn{10}{|l|}{ Modelo $3^{\star \star *}$} \\
\hline Unifatorial & 1,76 & 0,09 & 0,88 & 0,79 & 0,89 & 0,89 & 0,07 & 729,52 & 1,89 \\
\hline Tetrafatorial & 1,02 & 0,06 & 0,98 & 0,97 & 0,99 & 0,99 & $\begin{array}{c}0,01 \\
(0,00-0,04)\end{array}$ & 565,72 & $\begin{array}{c}1,37 \\
(1,35-1,52)\end{array}$ \\
\hline
\end{tabular}

Notas: *Modelo amostra total; **Modelo amostra jovens civis; ***Modelo amostra jovens militares.

Considerando que todas as saturações (Lambdas, $\lambda$ ) estiveram dentro do intervalo esperado $|0-1|$ e que foram estatisticamente diferentes de zero $(t>1,96$, $p<0,05)$, denotou-se, em relação à amostra total, não haver problemas de estimativa para corroborar a existência dos quatro fatores para se avaliar a empatia focada nos grupos. De forma geral, as associações entre as dimensões foram positivas, o que significa dizer que a existência de uma dessas formas de empatia, possivelmente, influenciará as outras formas. Na Figura 1, é possivel observar as associações entre as dimensões da empatia para amostra total.

$\mathrm{Na}$ Figura 2, pode-se observar a existência de lambdas $(\lambda)$ associativos, também positivos, entre as dimensões da empatia para a amostra de jovens civis. Note-se que esses resultados estão na mesma direção dos resultados da amostra geral.

Conforme pode ser visto na Figura 3, na amostra de jovens militares, as dimensões da empatia apresentaram lambdas $(\lambda)$ associados positivamente. Os resultados dessa amostra se assemelham aos encontrados na amostra geral e na dos jovens civis, e revelam uma consistência na mensuração da empatia na especificidade amostral desses sujeitos. 
FIGURA 1.

Estrutura Tetrafatorial da EEG, com a amostra total.

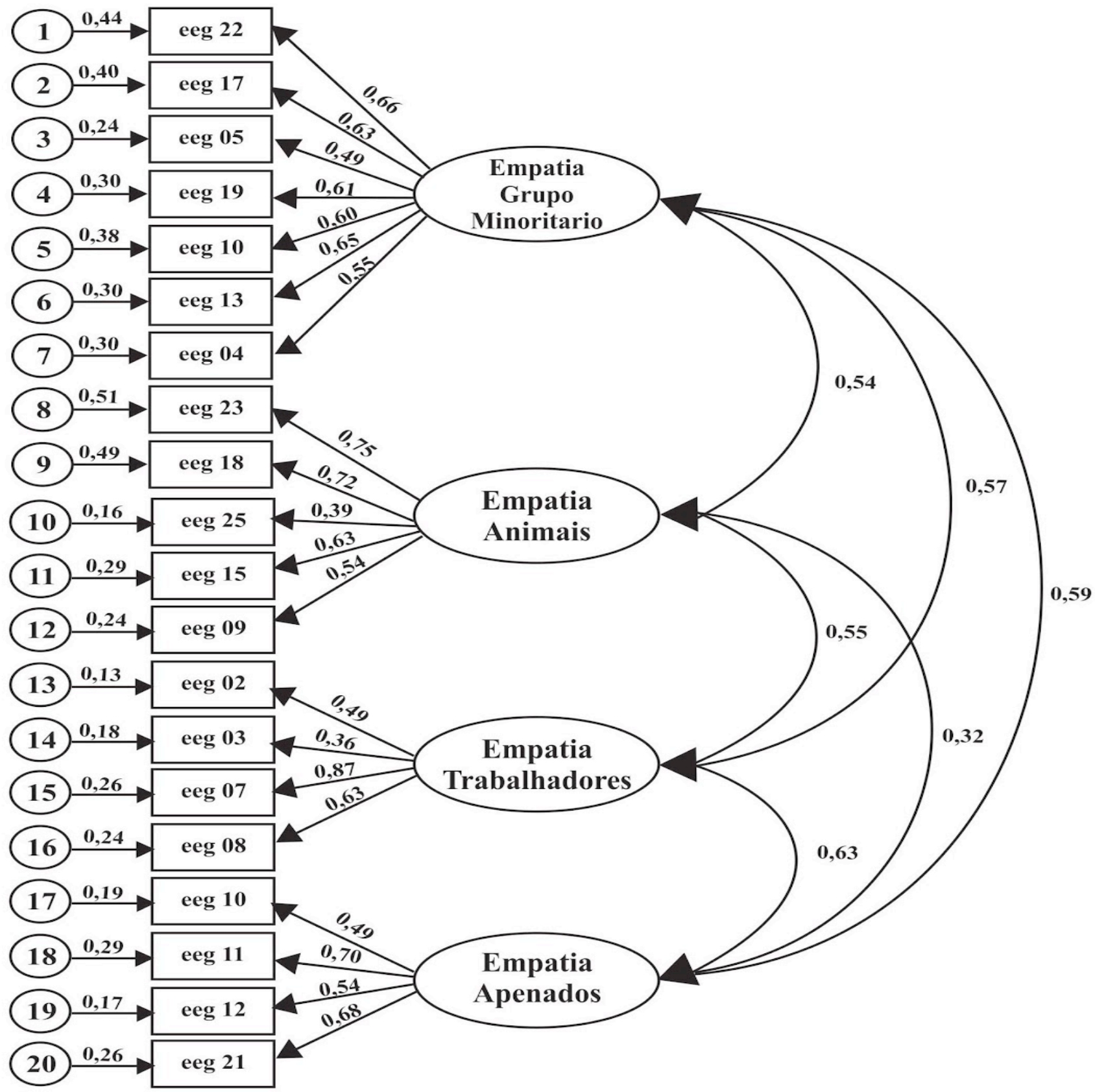


FIGURA 2.

Estrutura Tetrafatorial da EEG, com a amostra de jovens civis.

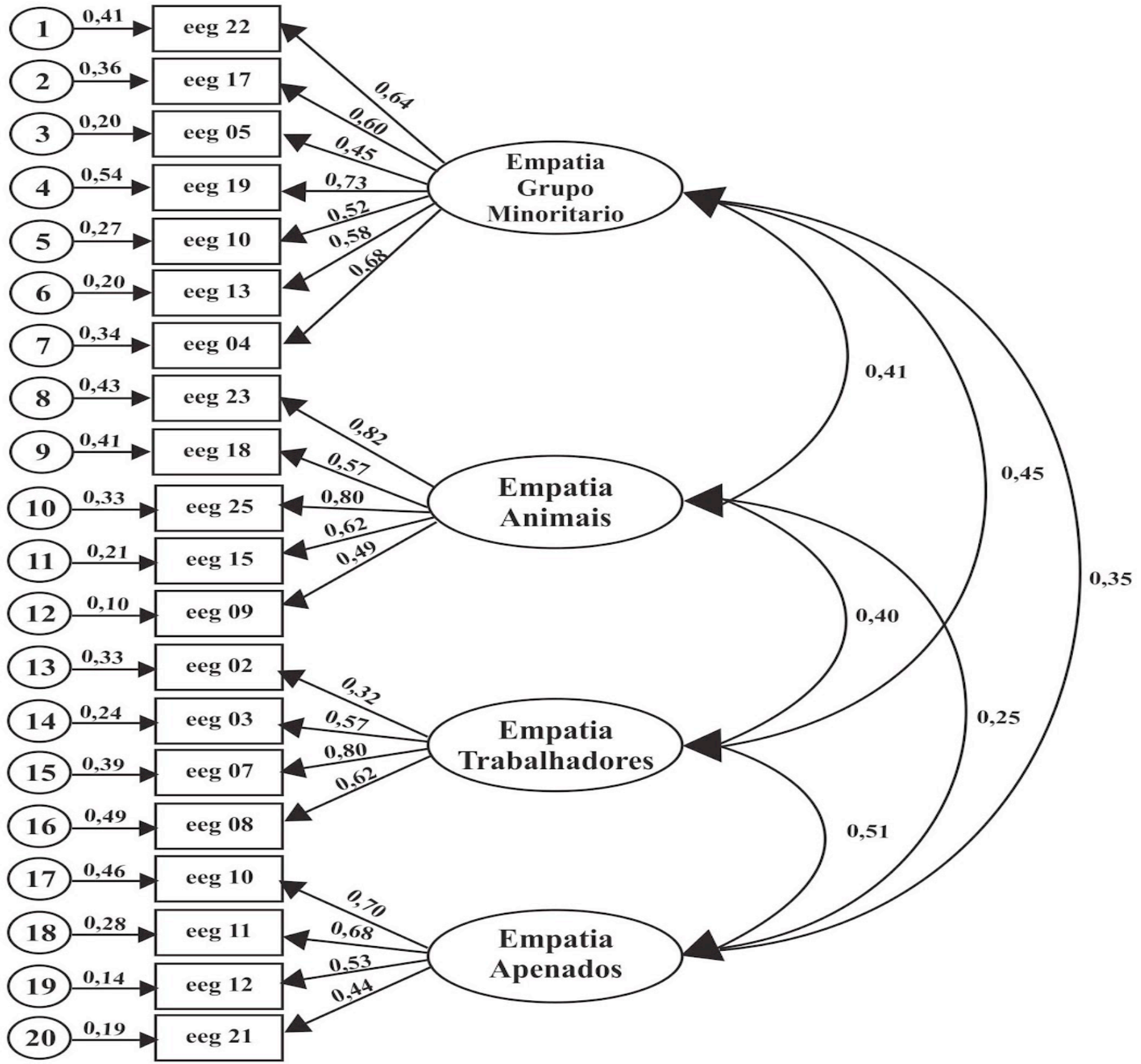


FIGURA 3.

Estrutura Tetrafatorial da EEG, com a amostra de jovens militares.

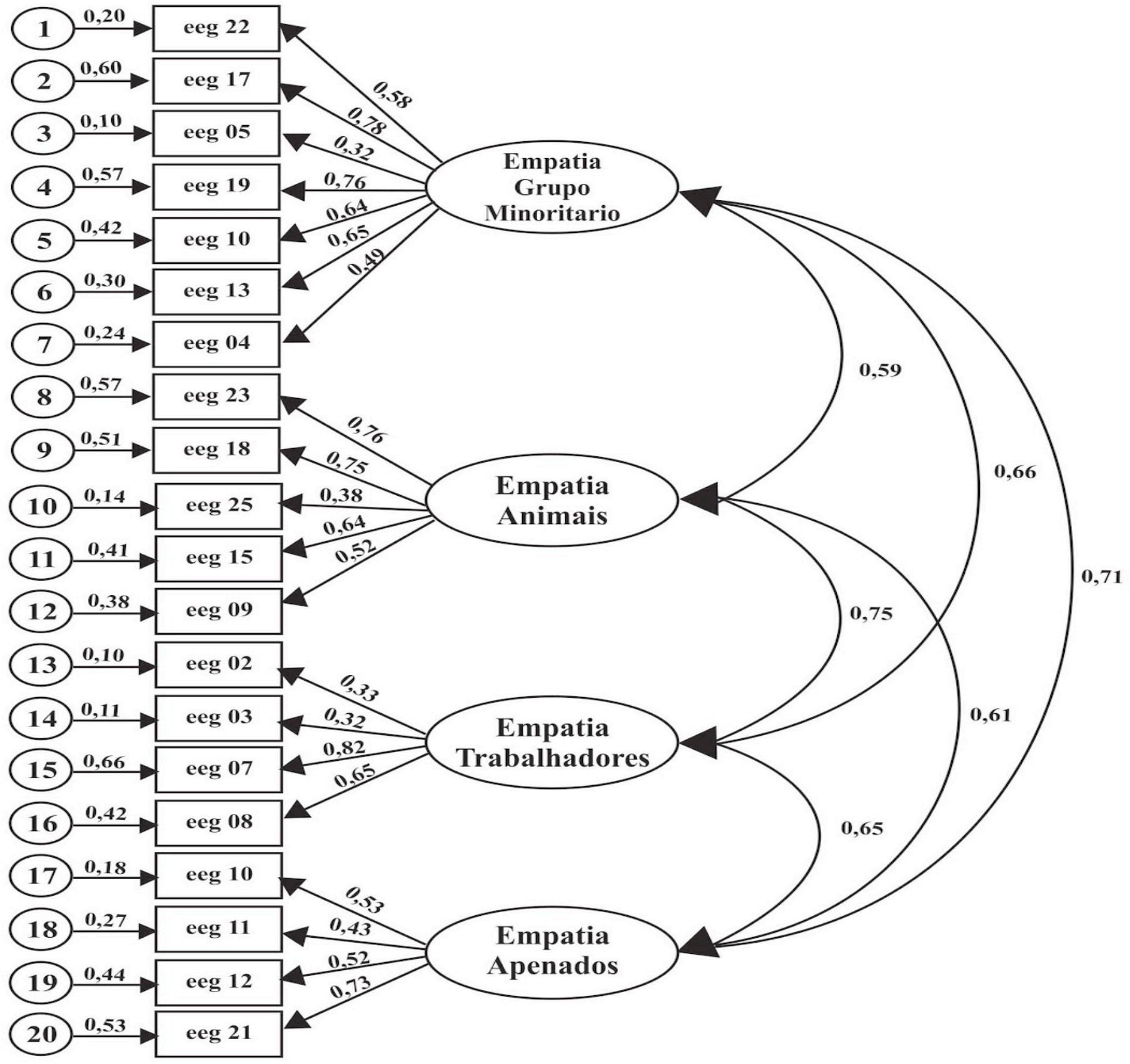


Note-se, que este modelo apresentou quatro componentes claramente discerníveis em relação aos grupos aos quais se destina o sentimento empático:

- Empatia com grupos minoritários, refere-se ao sentimento empático dirigido a grupos minoritários. $O$ fator é composto pelos itens: "crianças abusadas sexualmente" (eeg22), "negros sendo menosprezados" (eeg17), "pessoas doentes sem poder se tratar" (eeg05), "crianças sendo levadas à prostituição" (eeg19), "pessoas que são vítimas de catástrofes, como temor de terra" (eeg16), "pessoas vítimas de injustiça" (eeg13), "meninos pequenos usando drogas" (eeg 04).

- Empatia com animais em sofrimento, diz respeito ao sentimento empático dirigido a animais que estão sofrendo e/ou sendo maltratados. É composto pelos itens: "animais morrendo em período de seca" (eeg23), "animais sendo maltratados" (eeg18), "animais sendo levado ao matadouro" (eeg25), "animais em extinção" (eeg15), "animais sendo caçados como diversão das pessoas" (eeg09).

- Empatia com trabalhadores, refere-se ao sentimento empático direcionado a trabalhadores que estão sofrendo. É composto pelos itens: "pessoas que são obrigadas a deixar o campo para ir para cidade" (eeg08), "pessoas tendo que se levantar de madrugada para ir trabalhar" (eeg03), "pessoas trabalhando em serviços pesados mais de 12hs" (eeg07), "velhos pedindo esmolas" (eeg02)

- Empatia com apenados, relaciona-se ao sentimento empático dirigido a apenados que estão vivendo situações desumanas ou degradantes. É composto pelos itens: "pessoas condenadas à pena de morte por um crime que não cometeu" (eeg21), "os presos passarem muito tempo esperando julgamento" (eeg11), "presos vivendo em situações desumanas" (eeg12), "pessoas que cometeram crimes leves convivendo com pessoas que cometeram crimes graves" (eeg10).

É necessário salientar, no que se refere a validade deste construto, que realizaram-se, também, tanto o cálculo de confiabilidade composta (CC) quanto da variância média extraída (VME); no primeiro indicador exigese que o nível do escore seja acima de 0.70 , enquanto no segundo indicador é preciso um nível acima de 0.50 . Observouse que para as dimensões do EEG o CC e o VME, estiveram acima do exigido na literatura, isto é, observou-se, um CC que variou de 0.70 a 0.89 para as dimensões referentes as três organizações amostrais (isto é, a amostra geral, de jovens militares e jovens civis); em relação a VME observouse, também, para as três amostras uma variação de 0.77 a 1.00 . Tendo por base, tais indicadores, os resultados evidenciam a confiabilidade e validade convergente do construto utilizado. Desta forma, justifica-se a adequabilidade da estrutura fatorial do EGG, de forma confiável e com segurança fatorial para as referidas amostras.

Diante dos resultados obtidos, apresentados nas figuras 1, 2 e 3, assumese 0 modelo tetrafatorial da EEG como 0 mais adequado, tanto para a amostra geral, quanto para a amostra dos jovens civis e à amostra dos jovens militares, apoiando a hipótese levantada por Galvão et al. (2010) de que o modelo observado seria 0 mais adequado para avaliar esse tipo de empatia.

Comprovada a estrutura fatorial em distintas amostras procurou-se comparar, a partir de uma ANOVA, as pontuações médias das dimensões da empatia, considerando as instituições pesquisadas (civil $x$ militar). Foram encontrados os seguintes resultados: 
- em relação à empatia com grupos minoritários, os jovens militares apresentaram uma pontuação média superior (Média $=4,63, \mathrm{DP}=0,45 ; \mathrm{IC}-$ $4,57-4,70$ ) a dos jovens civis (Média $=$ $4,26$, DP $=0,63 ;$ IC $-4,17-4,35)[F$ $(1,381=43,49, p<0,01]$;

- no que se refere à empatia com trabalhadores, também, os jovens militares pontuaram mais alto (Média = $3,68, \mathrm{DP}=0,71 ; \mathrm{IC}-3,57-3,78$ ) do que os jovens civis (Média $=3,22$, DP $=$ $0,80 ; I C-3,11-3,38)[F(1,381=34,14$, $p<0,01$;

- a respeito da empatia com apenados, observou-se, também, que os sujeitos militares tiveram média superior (Média $=3,32$, DP = 0,88; IC - 3,19-3,45) em comparação com a dos jovens civis (Média $=2,79$, DP $=0,92 ; \mathrm{IC}-2,66$ $2,91)[F(1,381=33,62, p<0,01]$; em relação à dimensão empatia com animais, a diferença entre as pontuações médias dos jovens militares e civis não foi significativa.

\section{DISCUSSÃO}

De forma geral, em relação a escala EEG, os indicadores de bondade de ajuste (os quais, sugeridos pela literatura deste cálculo como garantia empírica [por exemplo, $X^{2} / g l$, GFI, AGFI, RMR, TLI, CFI, RMSEA, CAIC e $E C V I]$, apresentaram evidências da validade e robustez fatorial para 0 modelo fatorial, teoricamente proposto. Uma atenção dispensada a estes indicadores, poderá embasar tal medida do construto na referida amostra, bem como, de que os respondentes foram capazes de reconhecer a empatia focada em grupos e suas variações fatoriais, condição que corroborada pelo CC e VME.

Isto é, o respondente ao apresentar pontuações altas em uma das subdimensões estabelecidas para a medida do sentimento empático frente a grupos, provavelmente, terá escore médio alto nas demais subdimensões e na dimensão geral da EEG; sendo assim, justifica-se seu emprego no contexto de pesquisa com 0 objetivo de avaliar acerca de variáveis antecedentes e consequentes sobre 0 sentimento empático.

Considerando os resultados apresentando, no que se refere a amostra dos militares e ter, observado nesta, a existência de média superior à amostra dos jovens civis em três dos quatro componentes da empatia avaliados, julga-se que isto se deve a nova perspectiva social e educacional que é contemplada, na atualidade, aos jovens iniciados nas instituições de educação de segurança pública, pelo menos, é o que vem ocorrendo no Estado da Paraíba; merece destaque, na instituição militar pesquisada, a existência de um trabalho educacional com os jovens voltado para a Educação em Direitos Humanos e sensibilidade para a justiça social.

Entretanto, sobre a diferença supramencionada entre os jovens civis e os militares, podem ser levantados os seguintes questionamentos: (1) não teria essa diferença sido originada por outras características amostrais desses dois grupos, como o sexo e a idade? (2) não teria esta diferença ocorrido em função do que se espera socialmente de um jovem militar? Não estariam esses jovens sendo influenciados pela desejabilidade social? Esses questionamentos poderão ser respondidos em futuros estudos.

De forma geral, ao considerar o presente construto, acredita-se que o ser humano, independente do contexto escolar em que está inserido, é capaz de desenvolver um reconhecimento de uma situação-sujeito geradora da preocupação com o outro, desenvolvendo com isso, segundo Formiga, Rique, Galvão, Camino e Mathias (2011), uma espécie de ressonância interpessoal. Segundo esses autores, tal condição pressupõe que a pessoa que sente empatia 
é aquela que busca 0 respeito, a compreensão do outro e a participação no espaço sócio-cognitivo no campo dos problemas do outro. Nesse processo, aquele que pode ajudar dispõe de aberturas no espaço interpessoal afetivo, que the permite perceber a situação do outro, colocar-se no lugar do outro, sentir o que 0 outro sente e ter motivação para ajudá-lo.

É importante salientar que tanto 0 presente estudo como os desenvolvidos por Galvão et al. (2010) foram realizados com amostras brasileiras, o que significa que ao se considerar os resultados do presente estudo em outros contextos sociais e políticos, deve-se ter em conta as dimensões locais, específicas ou exclusivas - emics - da cultura em que os produziu, bem como, e não menos importante, as suas dimensões universais - etics (Muenjohn \& Armstrong, 2007; Triandis et. al., 1993; Van De Vijve \& Leung, 1997).

\section{CONCLUSÃO}

Espera-se que o objetivo deste estudo tenha sido alcançado, principalmente, no que diz a respeito à verificação da consistência e acurácia estrutural de uma escala de empatia focada em grupos. Assim, dispõe-se de mais um instrumento, relativamente confiável, para avaliar a empatia, operacionalizada de forma diferente de outras medidas empáticas. Isto é, em lugar de um instrumento de avaliação da capacidade empática de uma pessoa em relação a uma outra pessoa, o instrumento em pauta avalia a capacidade de uma pessoa empatizar com grupos de pessoas que se encontra em situações de sofrimento.

Deve-se, portanto, em próximos estudos, conhecer os aspectos que podem ser comuns a todas as culturas e aqueles que são específicos, 0 que contribuirá para consolidar um marco na teoria e na mensuração da empatia, já que, hipoteticamente, é possível encontrar variações desse construto ao considerar diferentes variáveis (como as sociodemográficas). Nessa direção, seria importante reunir evidências da validade e precisão intra, inter e pan-cultural, capaz de avaliar a validade (de critério ou a convergente) com construtos correlatos, bem como conhecer a estabilidade temporal (teste-reteste) e replicá-la com amostras maiores e diversificadas em relação às características dos participantes. 


\section{REFERÊNCIAS}

Associação nacional de pesquisa e pósgraduação em psicologia - ANPEPP (2011). Contribuições para a discussão das Resoluções CNS n. 196/96 e CFP $N^{\circ}$ 016/2000. Recuperado em 02 de Setembro de 2011, da WEB (página da WEB):

http://www.anpepp.org.br/XIISimposio/

Rel_ComissaoEticasobre_Res_CNS_e CFP.pdf2000

Beltrán, M. A. V. \& Cardona, M. A. (2005). La sociología frente a los espejos del tiempo: Modernidad, postmordernidad y globalización. Medelin: Universidad EAFIT.

Bilich, F., Silva, R. \& Ramos, P. (2006). Análise de flexibilidade em economia da informação: modelagem de equações estruturais. Revista de Gestão da Tecnologia e Sistemas de Informação, 3 (2), 93-122.

Byrne, B. M. (1989). A primer of LISREL: Basic applications and programming for confirmatory factor analytic models. New York: Springer-Verlag.

Byrne, B. M (2006). Structural Equation Modeling with EQS, basic concepts, applications, and programming. 2th edition, London: LEA Publishers.

Conselho Nacional de Saúde - CNS. (1996). Diretrizes e Normas Regulamentadoras de Pesquisas Envolvendo Seres Humanos. Recuperado em 02 de Setembro de 2011, da WEB (página da WEB): http://conselho.saude.gov.br/resolucoe s/reso_96.htm.

Davis, M. H. (1980). A multidimensional approach to individual differences in empathy. Catalog of Selected Documents in Psychology, 10 , 85, 117.

Dumont, L. (1985). O individualismo: Uma perspectiva antropológica da ideología moderna. Rio de Janeiro: Rocco.

Eisenberg, N. \& Strayer, J. (1990). Empathy and its development. New York: Cambridge University Press.
Formiga, N., Rique, J., Galvão, L., Camino, C. \& Mathias, A. (2011). Escala Multidimensional de Reatividade Interpessoal - EMRI: consistência estrutural da versal reduzida. Revista de Psicologia de Trujillo(Perú), 13 (2), 188-198.

Galvão, L., Camino, C., Gouveia, V. \& Formiga, N. (2010). Proposta de uma medida de empatia focada em grupos. PSICO, 41 (3), 399-405.

Hair, J. F., Anderson, R. E.; Tatham, R. L. \& Black, W. (2005). Análise Multivariada de Dados. Porto Alegre: Bookman.

Hoffman, M. L. (1991). Empathy, social cognition and moral action. Em: W. M. Kurtines \& J. L. Gewirtz (Orgs.), Handbook of moral behavior and development. Vol. 1. New Jersey: LEA.

Hoffman, M. L. (2003). Empathy and moral development:limplications for caring and justice. Cambridge, England: Cambridge University Press.

Hogan, R. (1969). Development of an Empathy Scale. Journal of Consulting and Clinical Psychology, 33, 307-316.

Joreskög, K. \& Sörbom, D. (1989). LISREL 7 user's reference guide. Mooresville: Scientific Software.

Lipovetsky, G. \& Charles, S. (2004). Os Tempos Hipermodernos. São Paulo: Barcarolla, Editorial Anagrama.

Mehrabian, A. \& Epstein, N. (1972). A measure of emotional empathy. Journal of Personality, 40, 525-543.

Morin, E. (2005) Educação e complexidade: Os sete saberes e outros ensaios. 3. ed. São Paulo: Editora Cortez.

Muenjohn, N. \& Armstrong, A. (2007). Transformational Leadership: The influence of culture on the leadership behaviors of expatriate managers. International Journal of Business and Information, 2, 265-283.

Oceja, L.V. \& Jiménez, I. (2007). Beyond egoism and group identity: Empathy toward the other and awareness of others in a social dilemma. The Spanish Journal of Psychology, 10 (2), 369-379. 
Paul, E. S. (2000). Empathy with animals and with humans: are they linked? Anthrozoos, 13(4), 194-202.

Pérez-Albeniz, A., De Paúl, J., Etxeberría, J., Montes, M. P., \& Torres, E. (2003). Adaptación de Interpersonal Reactivity Index (IRI) al español. Psicothema, 15, 267-272.

Schultz, D. P. \& Schultz, E. S. (1998). História da psicologia. São Paulo: Cultrix.

Strayer, J. \& Eisenberg, N. (1987). Empathy viewed in context. Em N. Eisenberg \& J. Strayer (Eds.), Empathy and its development (pp. 389-398). New York: Cambridge University Press.

Tabachnick, B. G. \& Fidell, L. S. (2001). Using multivariate statistics. California: Allyn \& Bacon.

Trianis, H. C., Mccusker, C., Betancourt, H., Iwao, S., Leung, K., Salazar, J. M., Setiadi, B., Sinha, B. P., Touzard, H. \& Zaleski, Z. (1993). Na etic-emic analysis of individualism and collectivism. Journal of crosscultural psychology, 24 (3), 366383.

Van De Vijver, F. \& Leung, K. (1997). Methods and data analysis for crosscultural research. Thousand Oaks, CA: Sage Publications.

Wispé, L. (1990). History of the concept of empathy. In: N. Eisenberg \& J. Strayer (org), Empathy and its development. (pp 17-37). New York: Cambridge University Press. 